\title{
Vallecular cysts in the pediatric population
}

\author{
An-Sofie Viskens ${ }^{1}$ (D), Nicolien Van der Poel ${ }^{1,2}$ (D), Stijn Verhulst ${ }^{1,3}$ (D), Vera Saldien ${ }^{1,4}$ (D), Olivier M. Vanderveken ${ }^{1,2}$ (D), An Boudewyns $\mathbf{S}^{1,2}$ (D) \\ ${ }^{1}$ Faculty of Medicine and Health Sciences, University of Antwerp, Antwerp, Belgium \\ 2Department of Otorhinolaryngology, Head and Neck Surgery, Antwerp University Hospital, Edegem, Belgium \\ ${ }^{3}$ Department of Pediatrics, Antwerp University Hospital, Edegem, Belgium \\ ${ }^{4}$ Department of Anesthesiology, Antwerp University Hospital, Edegem, Belgium
}

Cite this article as: Viskens AS, Van der Poel N, Verhulst S, Saldien V, Vanderveken OM, Boudewyns A. Vallecular cysts in the pediatric population. B-ENT 2021; 17(1): 42-44.

\begin{abstract}
Vallecular cysts (VCs) are a rare clinical entity in infants. In this report, we discuss 4 infants presenting with respiratory and feeding problems caused by VCs. The diagnosis was established by awake flexible endoscopy and was confirmed and treated using direct laryngoscopy. Marsupialization, with cold steel microsurgical instruments, was performed in all cases. General anesthesia proved to be challenging. Of the 4 cases, 3 had coexisting laryngomalacia that resolved spontaneously over time. Histopathology confirmed the benign character of the cyst. In general, postoperative course after marsupialization was favorable with no recurrence of VC and the initial symptoms.

Keywords: Vallecular cyst, stridor, infants, marsupialisation
\end{abstract}

\section{Introduction}

Vallecular cysts (VCs) are rare but may cause life-threatening airway obstruction requiring prompt diagnosis and treatment. We present a case series of 4 infants with VCs along with a review of the literature.

\section{Case Presentation}

All infants were born after uncomplicated pregnancies, and there were no medical problems at birth. Marsupialization of the anterior wall of the cyst was performed using cold steel instruments with the patient intubated under general anesthesia. Histopathological examination confirmed the benign nature of the cyst and normal epithelial lining.

Case 1 is of a 1-month-old male infant admitted because of noisy breathing and coughing during breastfeeding. Awake flexible endoscopy revealed a cystic mass in the vallecula, pushing the epiglottis against the posterior pharyngeal wall. A direct laryngoscopy (DL) under general anesthesia confirmed the diagnosis of VC. Intubation was complicated by the volume of the cyst, requiring the use of a laryngeal mask and fiberoptic guidance. The infant received postoperative respiratory support by high-flow nasal cannula (HFNC) for 1 day. Furthermore, postoperative course was favorable, with disappearance of the initial symptoms.
Case 2 is of a 1-month-old male infant admitted because of progressively worsening inspiratory stridor, feeding problems, and respiratory distress. A nasogastric tube was inserted, and respiratory support with HFNC was initiated. Polysomnography (PSG) demonstrated obstructive sleep apnea (OSA) with an obstructive apnea-hypopnea index (oAHI) of 7.8 events per hour. Therefore, cardiorespiratory monitoring (CRM) was started. Awake flexible endoscopy showed laryngomalacia, a large VC with secondary collapse of the epiglottis, and a retroposition of the tongue owing to retrognathia. DL confirmed these findings. Intubation was challenging owing to the volume of the VC and retrognathia. After a week, follow-up DL was performed, which still showed laryngomalacia but no recurrence of VC. Stridor and feeding problems improved and disappeared after a month.

Case 3 is of a 4-month-old female infant admitted to the pediatric ward because of inspiratory stridor, respiratory distress, feeding difficulties, and failure to thrive. Awake fiberendoscopy showed VC and laryngomalacia. The diagnosis was confirmed by DL. Successful intubation was achieved using a videolaryngoscope (Glidescope). On the first night postoperatively, she was admitted to the pediatric intensive care unit and supported by HFNC. The feeding difficulties resolved quickly, but the breathing difficulties persisted. PSG demonstrated an oAHI of 1.6 events per hour. CRM was started according to the clinical signs. The initial symptoms disappeared after the 1-month follow-up, and there was no recurrence of VC.

Corresponding Author: An Boudewyns, An.boudewyns@uza.be

Received: April 26, 2021 Accepted: November 20, 2021

Available online at www.b-ent.be

CC BY 4.0: Copyright@Author(s), "Content of this journal is licensed under a Creative Commons Attribution 4.0 International License." 


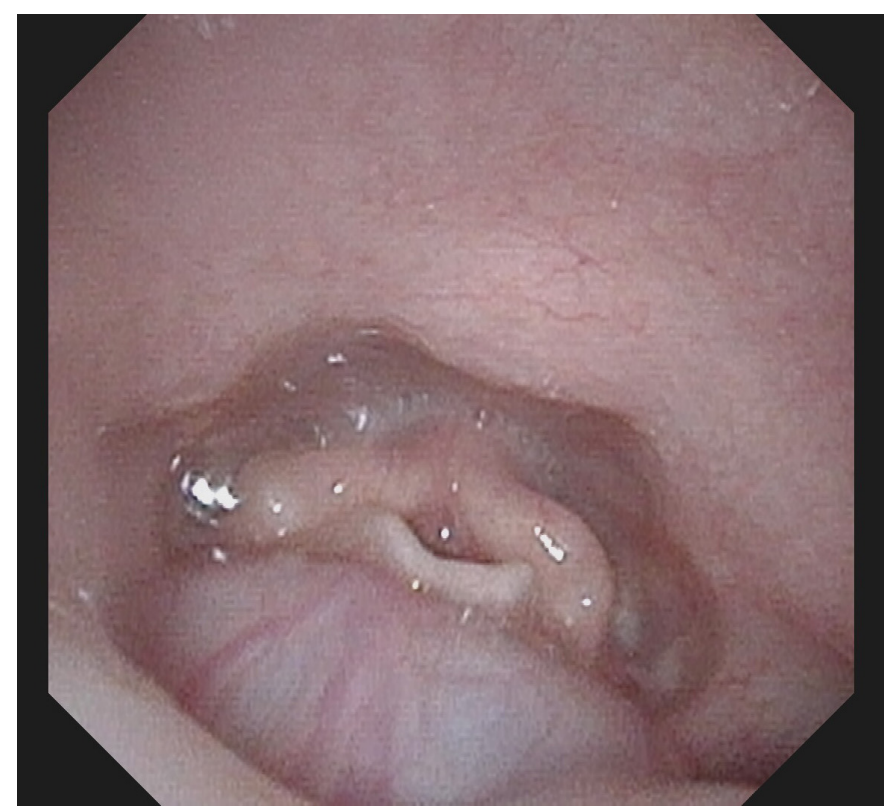

Figure 1. Awake flexible endoscopy showing the vallecular cyst, partially compressing the epiglottis, and obscuring the larynx

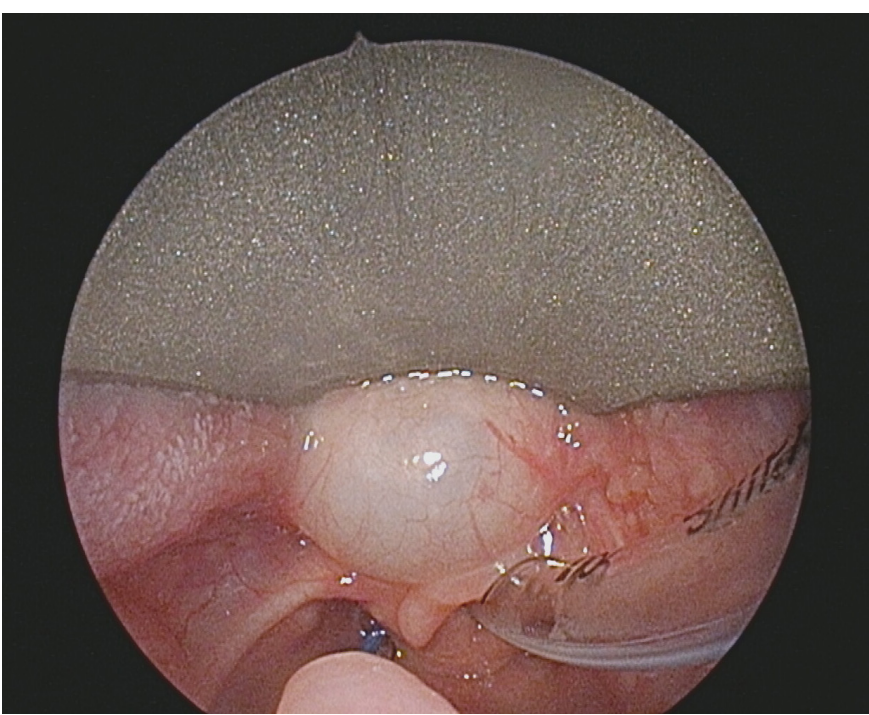

Figure 2. Direct laryngoscopy showing a vallecular cyst with secondary compression of the epiglottis

Case 4 is of a 2-week-old male infant referred from another hospital to our neonatal intensive care unit with a history of reduced intake and inspiratory stridor, evolving to respiratory fail-

\section{Main Points:}

Laryngomalacia frequently coexists with vallecular cyst (VC). Initial assessment with fiberendoscopy and confirmation by direct laryngoscopy (DL) are the diagnostic tools of choice.

Marsupialization by cold steel instruments leads to a quick improvement in the initial symptoms, and the symptoms have a very low recurrence rate.

Close cooperation with a pediatric anesthesiologist is required during $\mathrm{DL}$ as a difficult airway is more likely to occur when $\mathrm{VC}$ is present.

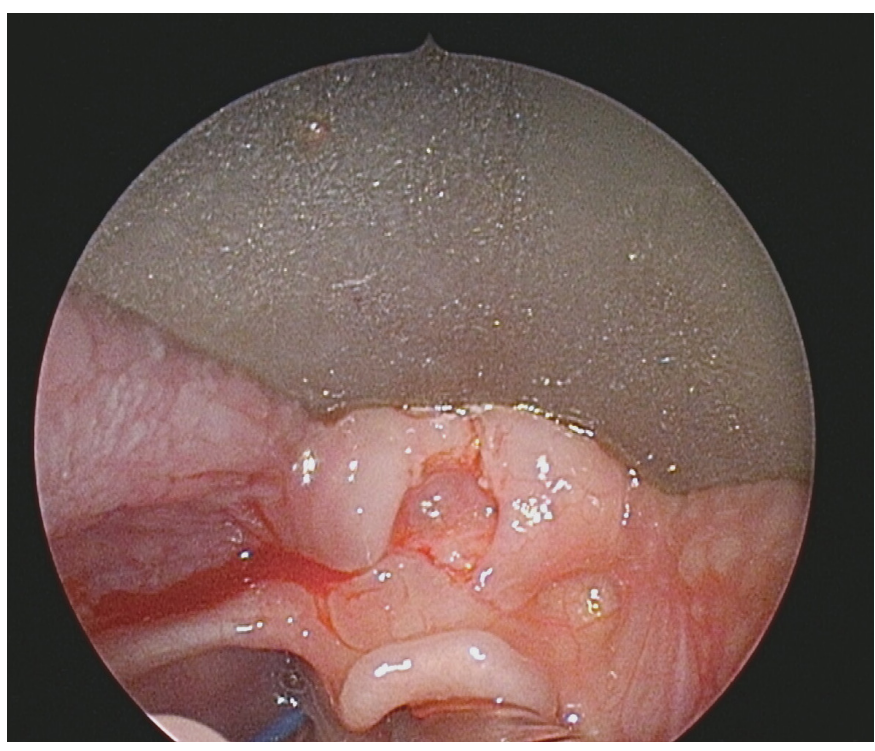

Figure 3. Direct laryngoscopy showing the vallecular cyst after marsupialisation. The anterior wall has been removed with cold steel microsurgical instruments

ure. Awake flexible endoscopy showed a mass in the pharynx behind the tongue, completely obscuring the larynx and suspicion of laryngomalacia (Figure 1). Treatment with continuous positive airway pressure (CPAP) was initiated. A direct laryngoscopy was performed under general anesthesia. Inhalation induction with sevoflurane was provided and spontaneous breathing was maintained. DL confirmed the diagnosis of VC, with secondary compression of the epiglottis, and laryngomalacia (Figure 2).

Regular intubation proved impossible and several attempts of fiberoptic intubation were required, which caused a hypoxic event to occur, before successfully intubating the patient. Figure 3 shows the cyst after marsupialization of the anterior wall. CPAP was continued the first postoperative day, and dexamethasone was administrated. Fiberendoscopy performed after a week still showed laryngomalacia. PSG was abnormal, with an oAHI of 33.6 events per hour. CRM was initiated and DL was repeated after a month. This showed no laryngomalacia and no recurrence of VC. The initial symptoms improved and did not reoccur. A PSG performed after 6 months showed mild OSA with an oAHI of 2.8 events per hour.

All procedures were performed in accordance with the ethical standards of the institutional research committee, and informed consent for the surgical procedures was obtained from the parents of the infants.

\section{Discussion}

We present 4 infants with increasing inspiratory stridor, feeding problems, and respiratory distress caused by VC. The progressive worsening of the symptoms differentiates this clinical entity from other more common causes of inspiratory stridor in infants, such as laryngomalacia.

$\mathrm{VC}$ is a rare cause of stridor and respiratory distress in newborns and infants (1-8). There is no gender preference (9). A bimodal age distribution suggests 2 clinical entities, either an adult or an infantile type, possibly being explained by different pathogen- 
esis. One theory suggests that VC is a consequence of a ductal obstruction of mucous glands, whereas another theory suggests that it is an embryological malformation (3). In neonates and young infants, owing to their relatively small airway, the cysts almost always cause an inspiratory stridor and may result in respiratory distress and possible life-threatening airway obstruction. Other common symptoms are feeding difficulties, episodes of cyanosis, and failure to thrive. The latter is more common if diagnosis is beyond the initial weeks of life (2). In our series, all cases presented with stridor and feeding difficulties, whereas failure to thrive was observed in the oldest patient (case 3).

In all cases, the diagnosis was established by awake flexible endoscopy and confirmed by DL under general anesthesia. We consider awake flexible endoscopy the best method for initial screening as it is minimally invasive and allows the recognition of VC. DL is required to confirm the diagnosis, and a surgical treatment can be performed under the same anesthesia that is administered during $\mathrm{DL}(1,7)$.

Laryngomalacia frequently coexists with VC and was documented in 3 of the 4 cases. Airway dynamics may be altered by the progressive enlargement of the cyst. This may increase inspiratory negative pressure, contributing to inspiratory collapse of supraglottic tissues and thus laryngomalacia (5). A posterior displacement of the laryngeal structures by the cyst may also aggravate the airway collapse during forceful inspiration $(4,8)$.

Differentiating between laryngomalacia and VC in cases of inspiratory stridor is very difficult without further endoscopic examination because of overlapping symptoms and frequent coexisting nature of both entities. Therefore, a high index of suspicion should be upheld with progressively worsening inspiratory stridor, feeding difficulties, failure to thrive, or progressively worsening symptoms of airway obstruction $(8,10)$.

OSA is another clinical entity in our cases. Marsupialization of VC may improve the obstructive breathing pattern, but this should be documented by PSG because OSA is a multifactorial condition in infants (11).

The primary treatment for VC is surgery. In all our cases, marsupialization of the VC using cold steel instruments was performed. Different techniques have been described using cold instruments or laser, complete excision, inside-out technique, and incision and drainage, depending on the surgeon's preference. Recurrence rate does not seem to be related to the surgical technique used (1). Only age and the presence of inflammation or infection appeared to influence the recurrence rate. The older the child, the more likely it is for VC to reoccur $(1,8)$.

Difficult intubation is a common feature of VC. In case 4 , a hypoxic event occurred during multiple attempts of fiberoptic intubation. Therefore, close collaboration with a pediatric anesthesiologist is required on suspicion of $\mathrm{VC}$, and a difficult airway should be anticipated.

The four cases presented in this report illustrate the typical features associated with vallecular cysts. Vallecular cysts are mostly symptomatic in newborns and infants and frequently coexist with laryngomalacia. Inspiratory stridor and feeding problems are the predominant symptoms, which are usually present in the first weeks of life. A high index of suspicion is required in case of progressively worsening inspiratory stridor along with signs of respiratory distress or growth failure. The diagnosis can be made by fiberendoscopy and confirmed by $\mathrm{DL}$, which allows surgical treatment of the cyst during the same anesthesia. Marsupialization with cold steel instruments is our preferred method of treatment. According to the literature, the recurrence rate is very low. Close cooperation with a pediatric anesthesiologist is required during $\mathrm{DL}$ because the volume of the cyst may compromise the airway and pose a challenge for intubation.

Informed Consent: Informed consent is not necessary due to the retrospective nature of this study.

Peer-review: Externally peer-reviewed.

Author Contributions: Supervision - A.B.; Design - A.V.; Resources N/A.; Materials - N/A.; Data Collection and/or Processing - A.B., A.V., N.V.D.P.; Analysis and/or Interpretation - A.B., A.V., N.V.D.P.; Literature Search - A.V.; Writing Manuscript A.V., A.B., N.V.D.P.; Critical Review S.V., V.S., O.M.V.

Conflict of Interest: The authors have no conflict of interest to declare.

Financial Disclosure: The authors declared that this study has received no financial support.

\section{References}

1. Leibowitz JM, Smith LP, Cohen MA, Dunham BP, Guttenberg M, Elden LM. Diagnosis and treatment of pediatric vallecular cysts and pseudocysts. Int J Ped Otorhinolaryngol 2011; 75: 899-904. [CrossRef]

2. Yao T-C, Chiu C-Y, Wu K-C, Wu L-J, Huang J-L. Failure to thrive caused by the coexistence of vallecular cyst, laryngomalacia and gastroesophageal reflux in an infant. Int J Ped Otorhinolaryngol 2004; 68: 1459-64. [CrossRef]

3. Gutiérrez JP, Berkowitz RG, Robertson CF. Vallecular cysts in newborns and young infants. Ped Pulmonol 1999; 27: 282-5. [CrossRef]

4. Ku A. Vallecular cyst: report of four cases - one with co-existing laryngomalacia. J Laryngol Otol 2000; 114: 224-6. [CrossRef]

5. Li $Y$, Irace AL, Dombrowski ND, Perez-Atayde AR, Robson CD, Rahbar R. Vallecular cyst in the pediatric population: Evaluation and management. Int J Ped Otorhinolaryngol 2018; 113: 198-203. [CrossRef]

6. Marseglia L, D'Angelo G, Impellizzeri $P$, et al. Neonatal stridor and laryngeal cyst: which comes first? Pediatrics Int 2017; 59: 115-7. [CrossRef]

7. Suzuki J, Hashimoto S, Watanabe K, Takahashi K. Congenital vallecular cyst in an infant: case report and review of 52 recent cases. J Laryngol Otol 2011; 12: 1199-203. [CrossRef]

8. Tsai Y-T, Lee L-A, Fang T-J, Li H-Y. Treatment of vallecular cysts in infants with and without coexisting laryngomalacia using endoscopic laser marsupialization: fifteen-year experience at a single-center. Int J Ped Otorhinolaryngol 2013; 77: 424-8. [CrossRef]

9. Faiss KR, Naji A, Sharma S. Anatomy, Head and neck, trachea epiglottic vallecula. StatPearls; 2020.

10. Hashemzadeh-Gargari R, Heslin SM, Sidlow R. Delayed diagnosis of an infected vallecular cyst in an infant. J Ped Surg Case Reports 2018; 38: 41-2. [CrossRef]

11. Kaditis AG, Alonso Alvarez ML, Boudewyns A, et al. ERS statement on obstructive sleep disordered breathing in 1- to 23-month-old children. Eur Respir J 2017; 50: 1700985. [CrossRef] 